\title{
Second and Secondary Lattice Modules
}

\author{
Fethi Çallıalp, ${ }^{1}$ Ünsal Tekir, ${ }^{2}$ Emel Aslankarayiğit Uğurlu, ${ }^{2}$ and Kürşat Hakan Oral ${ }^{3}$ \\ ${ }^{1}$ Department of Mathematics, Beykent University, Ayazaga-Maslak, 34396 Istanbul, Turkey \\ ${ }^{2}$ Department of Mathematics, Marmara University, Ziverbey, Göztepe, 34722 Istanbul, Turkey \\ ${ }^{3}$ Department of Mathematics, Yildiz Technical University, 34210 Istanbul, Turkey
}

Correspondence should be addressed to Kürşat Hakan Oral; khoral@yildiz.edu.tr

Received 22 April 2014; Accepted 7 September 2014; Published 14 October 2014

Academic Editor: Aldo Humberto Romero

Copyright (C) 2014 Fethi Çallıalp et al. This is an open access article distributed under the Creative Commons Attribution License, which permits unrestricted use, distribution, and reproduction in any medium, provided the original work is properly cited.

Let $M$ be a lattice module over the multiplicative lattice $L$. A nonzero $L$-lattice module $M$ is called second if for each $a \in L, a 1_{M}=1_{M}$ or $a 1_{M}=0_{M}$. A nonzero $L$-lattice module $M$ is called secondary if for each $a \in L, a 1_{M}=1_{M}$ or $a^{n} 1_{M}=0_{M}$ for some $n>0$. Our objective is to investigative properties of second and secondary lattice modules.

A multiplicative lattice $L$ is a complete lattice in which there is defined as a commutative, associative multiplication which distributes over arbitrary joins and has the compact greatest element $1_{L}$ (least element $0_{L}$ ) as a multiplicative identity (zero). An element $a \in L$ is said to be proper if $a<1_{L}$. An element $p<1_{L}$ in $L$ is said to be prime if $a b \leq p$ implies either $a \leq p$ or $b \leq p$. If $0_{L}$ is prime, then $L$ is said to be a domain. For $a \in L$, we define $\sqrt{a}=\bigvee\left\{x \in L: x^{n} \leq a\right.$ for some integer $\left.n\right\}$. An element $p<1_{L}$ in $L$ is said to be primary if $a b \leq p$ implies either $a \leq p$ or $b \leq \sqrt{p}$.

If $a, b$ belong to $L,\left(a::_{L} b\right)$ is the join of all $c \in L$ such that $c b \leq a$. An element $e$ of $L$ is called meet principal if $a \bigwedge b e=\left(\left(a:_{L} e\right) \bigwedge b\right) e$ for all $a, b \in L$. An element $e$ of $L$ is called join principal if $\left((a e \bigvee b):_{L} e\right)=a \bigvee\left(b:_{L} e\right)$ for all $a, b \in L . e \in L$ is said to be principal if $e$ is both meet principal and join principal. $e \in L$ is said to be weak meet (join) principal if $a \bigwedge e=e\left(a:_{L} e\right)\left(a \bigvee\left(0_{L}:_{L} e\right)=\left(e a:_{L} e\right)\right)$ for all $a \in L$. An element $a$ of a multiplicative lattice $L$ is called compact if $a \leq \bigvee b_{\alpha}$ implies $a \leq b_{\alpha_{1}} \bigvee b_{\alpha_{2}} \bigvee \cdots \bigvee b_{\alpha_{n}}$ for some subsets $\left\{\alpha_{1}, \alpha_{2}, \ldots, \alpha_{n}\right\}$. If each element of $L$ is a join of principal (compact) elements of $L$, then $L$ is called a $P G$ lattice (CG-lattice). If $L$ is a $C G$-lattice and $p$ is a primary element, then $\sqrt{p}$ is prime [1, Lemma 2.1].

Let $M$ be a complete lattice. Recall that $M$ is a lattice module over the multiplicative lattice $L$ or simply an $L$ module in case there is a multiplication between elements of $L$ and $M$, denoted by $l B$ for $l \in L$ and $B \in M$, which satisfies the following properties:
(1) $(l b) B=l(b B)$;

(2) $\left(\bigvee_{\alpha} l_{\alpha}\right)\left(\bigvee_{\beta} B_{\beta}\right)=\bigvee_{\alpha, \beta} l_{\alpha} B_{\beta}$;

(3) $1_{L} B=B$;

(4) $0_{L} B=0_{M}$;

for all $l, l_{\alpha}, b$ in $L$ and for all $B, B_{\beta}$ in $M$.

Let $M$ be an $L$-module. If $N, K$ belong to $M,\left(N:_{L} K\right)$ is the join of all $a \in L$ such that $a K \leq N$. Particularly, $\left(0_{M}:{ }_{L} 1_{M}\right)$ is denoted by $\operatorname{ann}(M)$. If $a \in L$ and $N \in M$, then $\left(N:_{M} a\right)$ is the join of all $H \in M$ such that $a H \leq N$. An element $N$ of $M$ is called meet principal if $\left(b \wedge\left(B:_{L} N\right)\right) N=b N \wedge B$ for all $b \in L$ and for all $B \in M$. An element $N$ of $M$ is called join principal if $b \vee\left(B:_{L} N\right)=\left((b N \vee B):_{L} N\right)$ for all $b \in L$ and for all $B \in M$. N is said to be principal if it is both meet principal and join principal. In special cases, an element $N$ of $M$ is called weak meet principal (weak join principal) if $\left(B:_{L} N\right) N=B \wedge N\left(\left(b N:_{L} N\right)=b \vee\left(0_{M}:_{L} N\right)\right)$ for all $B \epsilon$ $M$ (for all $b \in L$ ). $N$ is said to be weak principal if $N$ is both weak meet principal and weak join principal.

Let $M$ be an $L$-module. An element $N$ in $M$ is called compact if $N \leq \bigvee_{\alpha} B_{\alpha}$ implies $N \leq B_{\alpha_{1}} \vee B_{\alpha_{2}} \vee \cdots \vee B_{\alpha_{n}}$ for some subsets $\left\{\alpha_{1}, \alpha_{2}, \ldots, \alpha_{n}\right\}$. The greatest element of $M$ will be denoted by $1_{M}$. If each element of $M$ is a join of principal (compact) elements of $M$, then $M$ is called a $P G$ lattice module ( $C G$-lattice module).

Let $M$ be an $L$-module. An element $N \in M$ is said to be proper if $N<1_{M}$. For all elements $N$ of $M,\left[N, 1_{M}\right]$ is a set of 
all $K \in M$ such that $N \leq K \leq 1_{M}$ and $\left[N, 1_{M}\right]$ is an $L$-lattice module with $a \cdot K=a K \vee N$ for all $a \in L$ and $K \in M$ such that $N \leq K$.

For various characterizations of lattice modules, the reader is referred to [2-9].

Definition 1. A nonzero $L$-lattice module $M$ is called second if for each $a \in L, a 1_{M}=1_{M}$ or $a 1_{M}=0_{M}$.

Definition 2. A nonzero $L$-lattice module $M$ is called secondary if for each $a \in L, a 1_{M}=1_{M}$ or $a^{n} 1_{M}=0_{M}$ for some $n>0$.

Example 3. Let $Z$ be the integers, let $Q$ be the rational numbers, and let $Q$ be $Z$-module. Suppose $L=L(Z)$ is the set of all ideals of $Z$ and $M=L(Q)$ is the set of all submodules of $Q$. Thus, $M$ as $L$-lattice module is a second module, since for every integer $n \in Z,(n Z) Q=Q$ or $(n Z) Q=0$.

Remark 4. Every second lattice module is a secondary lattice. But the converse is not true. For this, we can give the following example.

Example 5. Let $Z$ be the integers and let $Z_{4}$ be $Z$-module. Suppose that $L=L(Z)$ is the set of all ideals of $Z$ and $M=$ $L\left(Z_{4}\right)$ is the set of all submodules of $Z_{4}$. Thus, $M$ as $L$-lattice module is a secondary lattice module, which is not a second lattice module.

Example 6. Let $Z$ be the integers and $L=L(Z)$ the set of all ideals of $Z$. Thus, $L$ as $L$-lattice module is neither a second lattice module nor a secondary lattice module.

Proposition 7. Let $L$ be a CG-lattice and let $M$ be a nonzero $L$-lattice module. If for each compact $a \in L, a 1_{M}=1_{M}$ or $a 1_{M}=0_{M}$, then $M$ is a second L-lattice module.

Proof. Let $r \in L$. Since $L$ is a CG-lattice, then we have $r=$ $\bigvee_{i} c_{i}$ such that $c_{i}$ 's are compact elements of $L$. Then, we obtain $r 1_{M}=\left(\bigvee_{i} c_{i}\right) 1_{M}=\bigvee_{i} c_{i} 1_{M}$. We have two cases.

Case 1. If $c_{i} 1_{M}=0_{M}$ for each compact $c_{i} \in L$, then we have $r 1_{M}=\left(\bigvee_{i} c_{i}\right) 1_{M}=\bigvee_{i} c_{i} 1_{M}=0_{M}$.

Case 2. If $c_{i} 1_{M}=1_{M}$ for some compact $c_{i} \in L$, then we have $r 1_{M}=\left(\bigvee_{i} c_{i}\right) 1_{M}=\bigvee_{i} c_{i} 1_{M}=1_{M}$.

Hence, $r 1_{M}=1_{M}$ or $r 1_{M}=0_{M}$ for each $r \in L$. Consequently, $M$ is second.

Proposition 8. If $M$ is a second L-lattice module, then $\operatorname{ann}(M)=\left(0_{M}:_{L} 1_{M}\right)=p$ is a prime element of $L$. In this case, $M$ is called $p$-second lattice module.

Proof. Suppose that $M$ is a second $L$-lattice module. Clearly, $\operatorname{ann}(M)=p$ is a proper element of $L$. Let $a b \leq p$ and assume that $b \nless p$; that is, $b 1_{M} \neq 0_{M}$. But $M$ is a second $L$-lattice module; then $b 1_{M}=1_{M}$. Since $b 1_{M}=1_{M}$ and $a b 1_{M}=0_{M}$, then $a 1_{M}=0_{M}$, which implies that $a \leq p$.
Proposition 9. If $M$ is a secondary L-lattice module, then $\operatorname{ann}(M)$ is a primary element of $L$.

Proof. Suppose that $M$ is a secondary $L$-lattice module. Let $a b \leq a n n(M)$ and $b \nless \sqrt{a n n(M)}$; we prove that $a \leq a n n(M)$. Since $a b \leq a n n(M)$ and $b \nless \sqrt{a n n(M)}$, we have $a b 1_{M}=0_{M}$ and $(b)^{n} 1_{M} \neq 0_{M}$ for each $n>0$. Since $M$ is secondary, we have $b 1_{M}=1_{M}$. Then $a b 1_{M}=a 1_{M}=0_{M}$, which implies $a \leq \operatorname{ann}(M)$.

Proposition 10. Let $L$ be a $C G$-lattice. If $M$ is a secondary $L$ lattice module, then $\sqrt{\operatorname{ann}(M)}=p$ is a prime element of $L$. In this case, $M$ is called p-secondary lattice module.

Proof. Let $M$ be a secondary lattice. Then $a n n(M)$ is a primary element of $L$ by Proposition 9. Therefore $\sqrt{\text { ann(M) }}$ is prime by [1, Lemma 2.1].

Proposition 11. Let $L$ be a lattice domain and let $M$ be a nonzero L-module. Then $M$ is a second lattice module with $\operatorname{ann}(M)=0_{L}$ if and only if $M$ is a secondary lattice module with $\sqrt{\operatorname{ann}(M)}=0_{L}$.

Proof. $\Rightarrow$ : Since $M$ is a second lattice module, then $M$ is a secondary lattice module. Since $L$ is domain, then $\sqrt{\operatorname{ann}(M)}=\sqrt{0_{L}}=0_{L}$.

$\Leftarrow$ : Suppose that $M$ is a secondary lattice module with $\sqrt{\operatorname{ann}(M)}=0_{L}$. Let $a \in L$ and assume that $a 1_{M} \neq 1_{M}$. Since $M$ is a secondary lattice module, then there exists a positive integer $n$ such that $a^{n} 1_{M}=0_{M}$; that is, $a \leq \sqrt{a n n(M)}=0_{L}$. Then $a 1_{M}=0_{M}$. Hence, we obtain $M$ is a second lattice. Clearly, $\operatorname{ann}(M)=0_{L}$.

Definition 12. Let $M$ be a nonzero $L$-lattice module. An element $0_{M} \neq N<1_{M}$ is said to be pure element, if $a N=$ $a 1_{M} \wedge N$ for all $a \in L$.

Proposition 13. Let $L$ be a CG-lattice, let $M$ be a nonzero $L$ lattice module, and let $N$ be a pure element of $M$. If $M$ is a $p$ secondary lattice module, then $\left[N, 1_{M}\right]$ and $\left[0_{M}, N\right]$ are both p-secondary lattice modules.

Proof. Suppose that $M$ is a $p$-secondary lattice module. Let $s \in L$. Since $M$ is a secondary lattice module, then either $s 1_{M}=1_{M}$ and in this case $s \cdot 1_{\left[N, 1_{M}\right]}=s \cdot 1_{M}=s 1_{M} \bigvee N=$ $1_{M} \bigvee N=1_{M}=1_{\left[N, 1_{M}\right]}$ or there exists a positive integer $t$, such that $s^{t} 1_{M}=0_{M}$ and in this case $s^{t} \cdot 1_{\left[N, 1_{M}\right]}=s^{t} \cdot 1_{M}=$ $s^{t} 1_{M} \bigvee N=0_{M} \bigvee N=N=0_{\left[N, 1_{M}\right]}$. Hence, $\left[N, 1_{M}\right]$ is a secondary lattice module.

It remains to show that $\sqrt{\operatorname{ann}\left(\left[N, 1_{M}\right]\right)}=p=\sqrt{\operatorname{ann}(M)}$. Clearly, $\sqrt{\operatorname{ann}(M)} \leq \sqrt{\operatorname{ann}\left(\left[N, 1_{M}\right]\right)}$. Let $r$ be compact and $r \leq \sqrt{\operatorname{ann}\left(\left[N, 1_{M}\right]\right)}$. Since $r$ is compact, there exists a positive integer $n$ such that $r^{n} \cdot 1_{\left[N, 1_{M}\right]}=0_{\left[N, 1_{M}\right]}$; that is, $r^{n} 1_{M} \bigvee N=N$. Hence, we have $r^{n} 1_{M} \leq N$. Now we assume that $r \nless \sqrt{\operatorname{ann}(M)}$. Then $r 1_{M}=1_{M}$, since $M$ is secondary. Thus, $1_{M}=r^{n} 1_{M} \leq N$; that is, $N=1_{M}$, which is a 
contradiction. Therefore, $r \leq \sqrt{\operatorname{ann}(M)}$. Consequently, we obtain $\sqrt{\operatorname{ann}\left(\left[N, 1_{M}\right]\right)} \leq \sqrt{\operatorname{ann}(M)}$.

Let $a \in L$. Since $N$ is pure, $a N=a 1_{M} \wedge N$. As $M$ is a secondary lattice module, then either $a 1_{M}=1_{M}$ or there exists a positive integer $n$ such that $a^{n} 1_{M}=0_{M}$. This implies that either $a N=N$ or $a^{n} N=a^{n} 1_{M} \wedge N=0_{M}$. Therefore, we have $a \cdot 1_{\left[0_{M}, N\right]}=a \cdot N=a N \bigvee 0_{M}=a N=N=1_{\left[0_{M}, N\right]}$ or $a^{n} \cdot 1_{\left[0_{M}, N\right]}=a^{n} \cdot N=a^{n} N \bigvee 0_{M}=a^{n} N=0_{M}=0_{\left[0_{M}, N\right]}$. Hence, $\left[0_{M}, N\right]$ is a secondary lattice module.

Now we show that $\sqrt{\operatorname{ann}(M)}=\sqrt{\operatorname{ann}\left(\left[0_{M}, N\right]\right)}$. Clearly, $\sqrt{\operatorname{ann}(M)} \leq \sqrt{\operatorname{ann}\left(\left[0_{M}, N\right]\right)}$. Let $c$ be compact and $c \leq$ $\sqrt{\operatorname{ann}\left(\left[0_{M}, N\right]\right)}$. Since $c$ is compact, there exists a positive integer $k$ such that $c^{k} \cdot N=c^{k} N=0_{\left[0_{M}, N\right]}=0_{M}$. Since $N$ is pure, we have $c^{k} N=c^{k} 1_{M} \wedge N=0_{M}$. If $c \nless \sqrt{\operatorname{ann}(M)}$, then $c 1_{M}=1_{M}$. Thus, $c^{k} 1_{M}=1_{M}$. This implies that $0_{M}=$ $c^{k} N=N \wedge c^{k} 1_{M}=N \wedge 1_{M}=N$, a contradiction. Therefore, $c \leq \sqrt{\operatorname{ann}(M)}$.

Proposition 14. Let $M$ be a nonzero L-lattice module and let $N$ be a pure element of $M$. Then $M$ is a p-second lattice module if and only if $\left[0_{M}, N\right]$ and $\left[N, 1_{M}\right]$ are both $p$-second lattice modules.

Proof. $\Rightarrow$ : Suppose that $M$ is a $p$-second lattice module. Let $a \in L$. Since $N$ is pure, we have $a N=N \bigwedge a 1_{M}$. As $M$ is a second lattice module, then either $a 1_{M}=0_{M}$ or $a 1_{M}=1_{M}$. This implies that either $a N=0_{M}$ or $a N=N$. Hence, $\left[0_{M}, N\right]$ is a second lattice module. Now, we show that $\operatorname{ann}(M)=$ $\operatorname{ann}\left(\left[0_{M}, N\right]\right)$. Clearly, $\operatorname{ann}(M) \leq \operatorname{ann}\left(\left[0_{M}, N\right]\right)$. Let $r \leq$ $\operatorname{ann}\left(\left[0_{M}, N\right]\right)$. Thus, we have $r N=0_{M}$. Now we assume that $r \$ \operatorname{ann}(M)$. Then we obtain $r 1_{M}=1_{M}$, since $M$ is a second lattice module. This implies that $0_{M}=r N=r 1_{M} \wedge N=$ $1_{M} \wedge N=N$, a contradiction. Therefore, $r \leq \operatorname{ann}(M)$.

Let $t \in L$. Since $M$ is a second lattice module, either $t 1_{M}=1_{M}$ and in this case $t \cdot 1_{\left[N, 1_{M}\right]}=t \cdot 1_{M}=t 1_{M} \bigvee N=$ $1_{M} \bigvee N=1_{M}=1_{\left[N, 1_{M}\right]}$ or $t 1_{M}=0_{M}$ and in this case $t$. $1_{\left[N, 1_{M}\right]}=t \cdot 1_{M}=t 1_{M} \bigvee N=0_{M} \bigvee N=N=0_{\left[N, 1_{M}\right]}$. Hence, $\left[N, 1_{M}\right]$ is a second lattice module. It remains to show that $\operatorname{ann}(M)=\operatorname{ann}\left(\left[N, 1_{M}\right]\right)$. Clearly $\operatorname{ann}(M) \leq \operatorname{ann}\left(\left[N, 1_{M}\right]\right)$. Let $s \leq \operatorname{ann}\left(\left[N, 1_{M}\right]\right)$. Thus, $s \cdot 1_{\left[N, 1_{M}\right]}=0_{\left[N, 1_{M}\right]}$; that is, $s 1_{M} \bigvee N=N$, and this implies that $s 1_{M} \leq N$. Now we suppose that $s \nless$ ann $(M)$. Then, we have $s 1_{M}=1_{M}$, since $M$ is second. Hence, $1_{M}=s 1_{M} \leq N$, a contradiction. Therefore, $s \leq \operatorname{ann}(M)$.

$\Leftarrow$ : Suppose that $\left[0_{M}, N\right]$ and $\left[N, 1_{M}\right]$ are both second lattice modules with $\operatorname{ann}\left(\left[0_{M}, N\right]\right)=\operatorname{ann}\left(\left[N, 1_{M}\right]\right)=p$. Let $r \in L$. We have two cases.

Case 1. If $r \leq \operatorname{ann}\left(\left[0_{M}, N\right]\right)=\operatorname{ann}\left(\left[N, 1_{M}\right]\right)$, then $r N=0_{M}$ and $r \cdot 1_{\left[N, 1_{M}\right]}=r \cdot 1_{M}=r 1_{M} \bigvee N=N$, which implies $r 1_{M} \leq N$. Thus, $0_{M}=r N=N \bigwedge r 1_{M}=r 1_{M}$.

Case 2. If $r \nless \operatorname{ann}\left(\left[0_{M}, N\right]\right)=\operatorname{ann}\left(\left[N, 1_{M}\right]\right)$, then $r N=N$ since $\left[0_{M}, N\right]$ is a second lattice module. Hence, we have $N=$ $r N=N \wedge r 1_{M}$, that is, $N \leq r 1_{M}$, since $N$ is pure. Because $\left[N, 1_{M}\right]$ is a second lattice module and $r \nless \operatorname{ann}\left(\left[N, 1_{M}\right]\right)$; we obtain $r \cdot 1_{\left[N, 1_{M}\right]}=r 1_{M} \bigvee N=1_{\left[N, 1_{M}\right]}=1_{M}$. Therefore, we obtain that $r 1_{M}=1_{M}$. Consequently, $M$ is a second lattice module.

Now we show that $\operatorname{ann}(M)=p$. Clearly $\operatorname{ann}(M) \leq$ $\operatorname{ann}\left(\left[N, 1_{M}\right]\right)$. Let $s \leq \operatorname{ann}\left(\left[N, 1_{M}\right]\right)$. Then we have $s \cdot 1_{\left[N, 1_{M}\right]}=$ $0_{\left[N, 1_{M}\right]}$, that is; $s \cdot 1_{M}=N$. Thus, $s 1_{M} \bigvee N=N$, and so $s 1_{M} \leq N$. Now, we assume that $s \nless \operatorname{ann}(M)$. Then, we have $s 1_{M}=1_{M}$, since $M$ is second. Hence, $1_{M}=s 1_{M} \leq N$, a contradiction. Consequently, we have $s \leq \operatorname{ann}(M)$.

Definition 15. An $L$-module $M$ is called a multiplication lattice module if for every element $N \in M$, there exists an element $a \in L$, such that $N=a 1_{M}$.

Definition 16. A element $N$ of an $L$-module $M$ is called prime element if $N \neq 1_{M}$ and whenever $r \in L$ and $X \in M$ with $r X \leq N$, then $X \leq N$ or $r \leq\left(N:_{L} 1_{M}\right)$.

Definition 17. A element $N$ of an $L$-module $M$ is called semiprime element if $N \neq 1_{M}$ and whenever $r \in L$ and $X \in M$ with $r^{2} X \leq N$, then $r X \leq N$.

Remark 18. Let $N$ be a proper element of an $L$-module $M$. Then $N$ is a semiprime element if and only if whenever $r \in L$, $X \in M$ and $k$ is a positive integer with $r^{k} X \leq N$, then $r X \leq$ $N$.

We know that a prime element is semiprime, but the converse is not true in general. The following proposition shows that the converse is true when the module is secondary and multiplication.

Proposition 19. Let $M$ be a multiplication and secondary $L$ lattice module. For all element $N$ of $M$ such that $1_{M} \neq N \in$ $M, N$ is a semiprime element of $M$ if and only if $N$ is a prime element of $M$.

Proof. $\Rightarrow$ : Suppose that $N$ is a semiprime element of $M$ and let $r X \leq N$, where $r \in L, X \in M$. Since $M$ is a secondary lattice module, then either $r^{n} 1_{M}=0_{M}$ for some positive integer $n$ or $r 1_{M}=1_{M}$.

Case 1. If $r^{n} 1_{M}=0_{M}$, then $r^{n} 1_{M} \leq N$. Since $N$ is a semiprime element, we have $r 1_{M} \leq N$.

Case 2. If $r 1_{M}=1_{M}$, then we have $X=r X$, since $M$ is a multiplication lattice module. Then we have $r X=X \leq N$.

Therefore, $N$ is a prime element of $M$.

$\Leftarrow$ : It is obvious.

Definition 20. Let $M$ be an $L$-lattice module and let $N$ be a proper element of $M . N$ is called a primary element of $M$, if whenever $a \in L, X \in M$ such that $a X \leq N$, then $X \leq N$ or $a \leq \sqrt{\left(N:_{L} 1_{M}\right)}$. Particularly, if $M$ is nonzero and $0_{M}$ is primary, then $M$ is said to be primary lattice module.

Definition 21. An $L$-lattice module $M$ is said to be simple lattice module if $M=\left\{0_{M}, 1_{M}\right\}$. 
Proposition 22. Every multiplication secondary lattice module is a primary lattice module.

Proof. Let $M$ be a multiplication secondary module and $r X=$ $0_{M}$ for some $r \in L, X \in M$. Now, we assume that $r \sqrt{\operatorname{ann}(M)}$. Since $M$ is a secondary module, then we have $r 1_{M}=1_{M}$. Because $M$ is a multiplication, then we have $r X=X$. Consequently, we obtain $X=0_{M}$.

Proposition 23. Every multiplication second lattice module is a simple lattice module.

Proof. Let $M$ be a multiplication and second module. Since $M$ is a multiplication, for every $N \in M$, there exists $a \in L$ such that $N=a 1_{M}$. Then we obtain $a 1_{M}=1_{M}$ or $a 1_{M}=0_{M}$, since $M$ is second. Thus, we have $N=1_{M}$ or $N=0_{M}$ for every $N \in M$; that is, $M$ is simple.

Definition 24. Let $L$ be a domain and let $M$ be a nonzero $L$ lattice module. If $r 1_{M}=1_{M}$ for every $0_{L} \neq r \in L$, then $M$ is said to be divisible.

Definition 25. A nonzero $L$-lattice module $M$ is said to be torsion if there exists $0_{L} \neq r \in L$ such that $r 1_{M}=0_{M}$.

Proposition 26. Let $L$ be a domain. Let $M$ be a secondary $L$ lattice module. Then either $M$ is a divisible module or $M$ is a torsion module.

Proof. Suppose that $M$ is a secondary module over a domain $L$. If $M$ is not divisible, then there exists $0_{L} \neq r \in L$ such that $r 1_{M} \neq 1_{M}$. Since $M$ is a secondary lattice module, then there exists a positive integer $n$ such that $r^{n} 1_{M}=0_{M}$. Since $0_{L} \neq r$ and $L$ is a domain, then we have $r^{n} \neq 0_{L}$. Consequently, there exists $0_{L} \neq r^{n}=s \in L$ such that $s 1_{M}=0_{M}$. Therefore, $M$ is a torsion lattice module.

\section{Conflict of Interests}

The authors declare that there is no conflict of interests regarding the publication of this paper.

\section{References}

[1] J. A. Johnson, "The structure of a class of $r$-lattices," Commentarii Mathematici Universitatis Sancti Pauli, vol. 32, no. 2, pp. 89-94, 1983.

[2] F. Callialp and U. Tekir, "Multiplication lattice modules," Iranian Journal of Science and Technology, vol. 35, no. 4, pp. 309-313, 2011.

[3] E. W. Johnson and J. A. Johnson, "Lattice modules over principal element domains," Communications in Algebra, vol. 31, no. 7, pp. 3505-3518, 2003.

[4] D. S. Culhan, Associated Primes and Primal Decomposition in Modules and Lattice Modules, and Their Duals, University of California Riverside, 2005.

[5] E. A. Al-Khouja, "Maximal elements and prime elements in lattice modules," Damascus University for Basic Sciences, vol. 19, pp. 9-20, 2003.
[6] H. M. Nakkar, "Localization in multiplicative lattice modules," Istoriko-Matematicheskie Issledovaniya, vol. 2, no. 32, pp. 88108, 1974 (Russian).

[7] H. M. Nakkar and I. A. Al-Khouja, "Multiplication elements and distributive and supporting elements in lattice modules," Research Journal of Aleppo University, vol. 11, pp. 91-110, 1989.

[8] H. M. Nakkar and I. A. Al-Khouja, "Nakayama's Lemma and the principal elements in Lattice Modules over multiplicative lattices," Research Journal of Aleppo University, vol. 7, pp. 1-16, 1985.

[9] H. M. Nakkar and D. D. Anderson, "Associated and weakly associated prime elements and primary decomposition in lattice modules," Algebra Universalis, vol. 25, no. 2, pp. 196-209, 1988. 


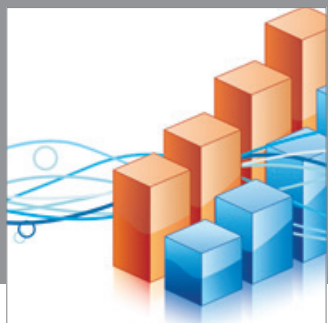

Advances in

Operations Research

mansans

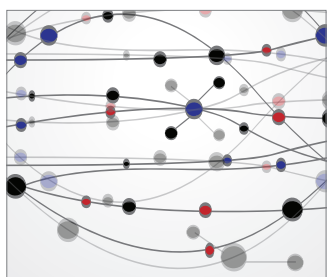

The Scientific World Journal
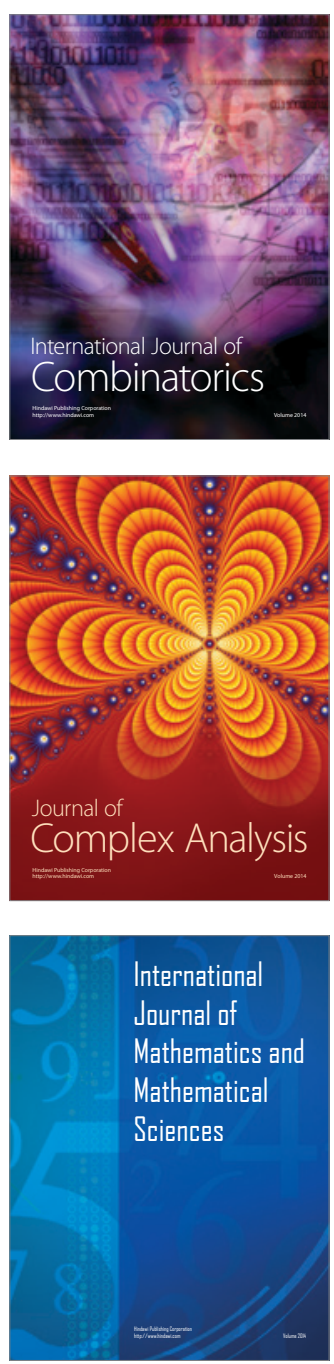
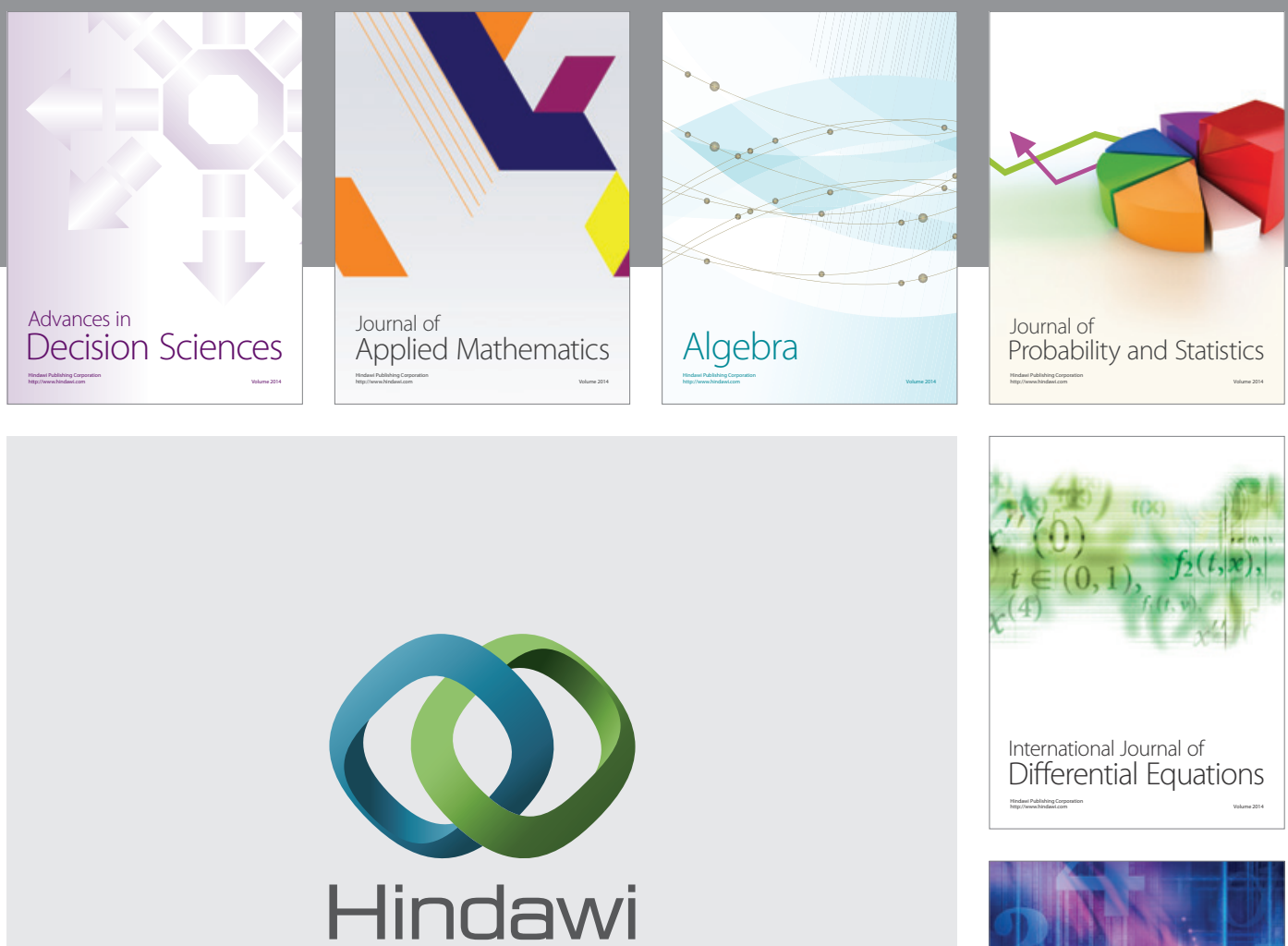

Submit your manuscripts at http://www.hindawi.com
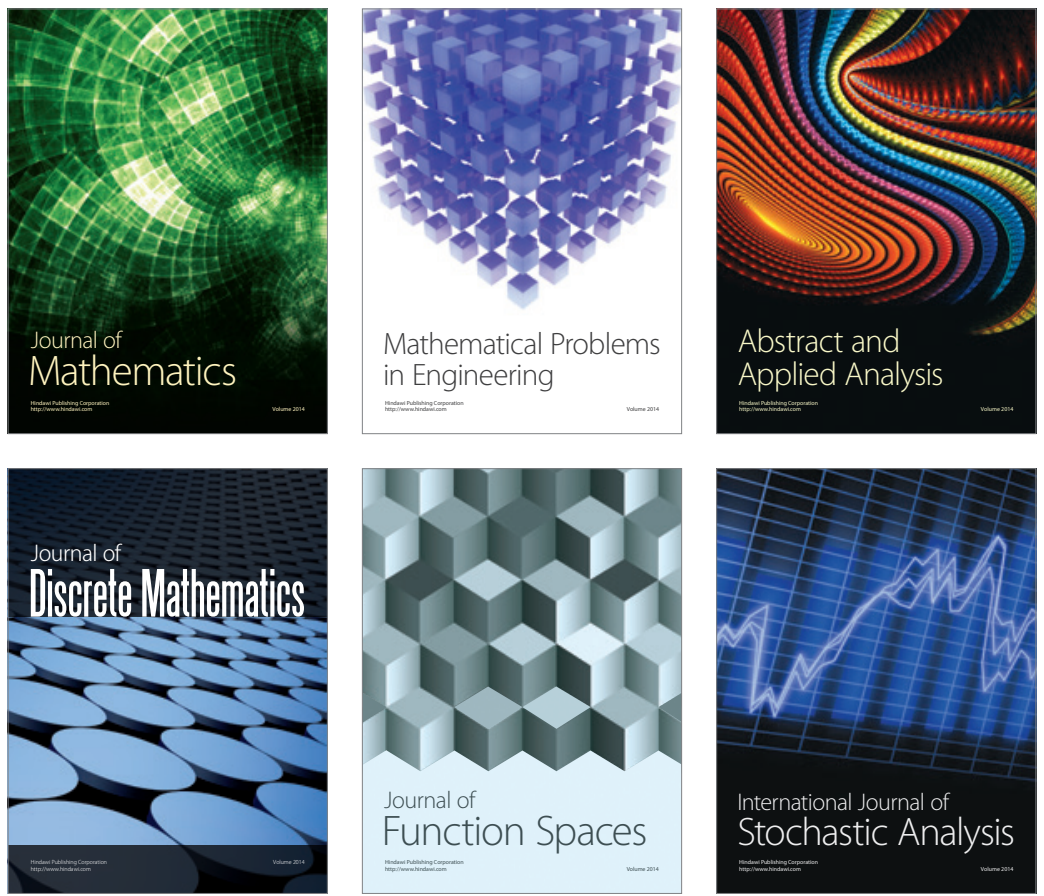

Journal of

Function Spaces

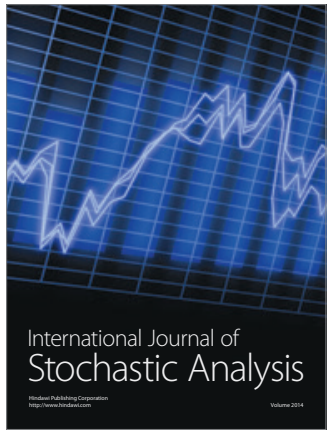

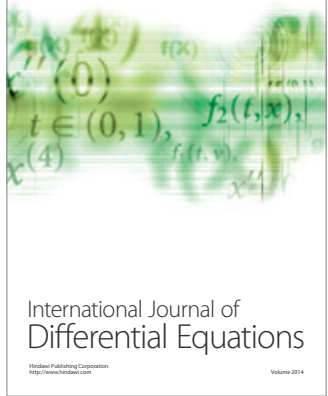
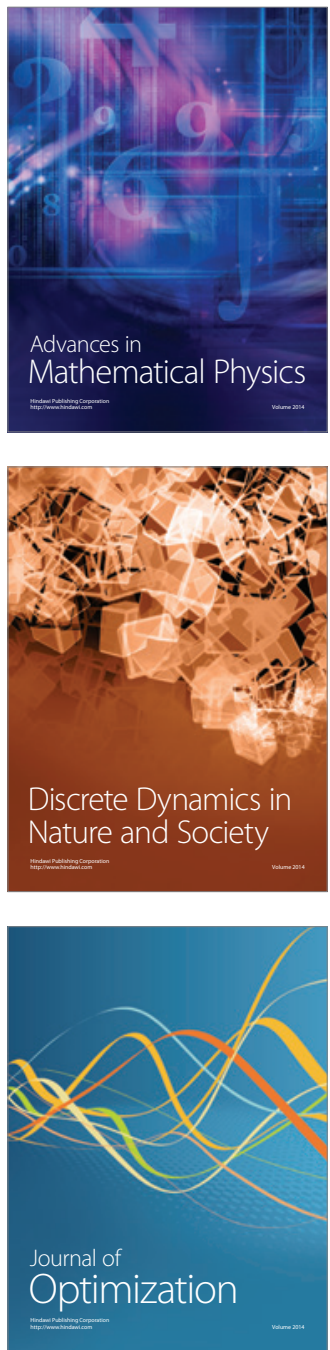